\title{
Determination of dynamic fracture toughness using a new experimental technique
}

\author{
Carl M. Cady ${ }^{\mathrm{a}}$, Cheng Liu, and Manuel L. Lovato \\ MST-8, MS-G755, Los Alamos National Laboratory, Los Alamos NM 87545, USA
}

\begin{abstract}
In other studies dynamic fracture toughness has been measured using Charpy impact and modified Hopkinson Bar techniques. In this paper results will be shown for the measurement of fracture toughness using a new test geometry. The crack propagation velocities range from $\sim 0.15 \mathrm{~mm} / \mathrm{s}$ to $2.5 \mathrm{~m} / \mathrm{s}$. Digital image correlation (DIC) will be the technique used to measure both the strain and the crack growth rates. The boundary of the crack is determined using the correlation coefficient generated during image analysis and with interframe timing the crack growth rate and crack opening can be determined. A comparison of static and dynamic loading experiments will be made for brittle polymeric materials. The analysis technique presented by Sammis et al. [1] is a semi-empirical solution, however, additional Linear Elastic Fracture Mechanics analysis of the strain fields generated as part of the DIC analysis allow for the more commonly used method resembling the crack tip opening displacement (CTOD) experiment. It should be noted that this technique was developed because limited amounts of material were available and crack growth rates were to fast for a standard CTOD method.
\end{abstract}

\section{Introduction}

The primary reason for this investigation was to determine the most accurate means of measuring the effect of aging in polymeric materials and polymer bonded composites. It is strongly believed that fracture toughness is the property that is most sensitive to chemical and microstructural changes that occur in these materials. Initial characterization was pursued using several common techniques including 3 and 4 point bend tests, Brazil tests, and Brazil tests with initial damage [2-6]. Each of these test geometries were non-ideal because the crack growth rates were not easily controlled and there was significant variability in test results. Finally, the "compressionfracture" test geometry introduced by Sammis et al. [1] was settled on because the crack growth rate can be easily controlled using the loading rate and repeat tests show minimal sample to sample variation. In addition to the fracture toughness tests, simple tensile tests were performed to find Poisson's ratio and the stiffness modulus. Initially, the semi-empirical equation introduced by Sammis et al. [1] for fracture toughness was used to generate a curve for the fracture toughness. Evaluation of the deformation data indicated that a linear elastic fracture mechanics (LEFM) solution could be used to find the unique solution for fracture toughness. The materials studied for this research were Diallyl Phthalate (DAP), a rigid polymer having good dimensional stability and impact resistance, and an epoxy bonded ceramic (EBC). The binder content of the EBC is $10 \mathrm{wt} \%$ $(\sim 22$ vol. \%) with a ceramic size distribution varying from 10 to $350 \mu \mathrm{m}$. The materials have little strain to failure; therefore, lubrication was assumed to be unnecessary.

\footnotetext{
${ }^{a}$ Corresponding author: cady@lanl .gov
}

\section{Experimental methods}

Mechanical testing was conducted on an Instron 1125 electro-mechanical load frame with a MTS "renew" control package for slow loading rate tests and on an MTS 810 servo-hydraulic system with a cross-head speed up to $4.5 \mathrm{~m} / \mathrm{s}$ for the dynamic experiments. For the low rate tests a sub press was used to ensure axial loading and minimize the risk of off axis deflection. No lubrication was used at the loading surfaces between the frame and the sample but optically flat platens were used as the contact surface. For the high rate tests the sample was bonded to the fixed side of the load chain using vacuum grease. Because failure was seen to occur at small displacements and is caused by tensile crack growth, the increased friction was assumed to have little influence on the test results. As in the low rate tests the unconstrained end of the sample was unlubricated. Experimental data are captured simultaneously on the load frame controller and on an image capture system for the low rate tests. At high rates, contact and failure images were synchronized with the load-displacement data so that the images could be matched to corresponding loads.

The characterization technique used to measure displacement and strain in these experiments is the digital image correlation (DIC). DIC has been demonstrated to be robust, flexible, applicable to large deformation and over a wide range of size scales and very affordable (as sophisticated optics instrumentation is usually not required). The underlying principle of digital image correlation as a deformation measurement technique is rather simple. It relies on the computer vision approach to extract the whole-field displacement data, that is, by comparing the features in a pair of digital images of a specimen surface before and after deformation. Since the displacements are calculated directly by correlating 
the two digital images, processing of fringe patterns or other secondary phenomena to yield deformations is not required. Details of standard DIC analysis are described elsewhere [7].

The advantage to using DIC is that the images can be used to look at both the global strain and local strain, and it is possible to reanalyze the data in many different ways. This means that it is possible to get a large amount of information from a single experiment. The DIC technique can detect motion as small as 0.02 pixels. As a result, if the gage section is 100 pixels across in the image, the accuracy of strain measurement will be $0.02 \%$, assuming the deformation in the gage section is uniform. The larger the image size the higher accuracy of measurement can achieve. Since the strain from the DIC technique does not have any machine compliance, it very accurately shows the early loading stages. It is essentially like putting a strain gage of variable size or multiple strain gages on the test specimen. Further details on the stress analysis for the "compression-fracture" geometry will be described in the section on sample geometry.

Another key characteristic of the DIC technique is the ability to detect a breakdown in image quality. For the analysis conducted in this study this is a critical capability of the software provided. This parameter is known as the correlation coefficient. The definition of a crack in this study is defined as the value when "image correlation" begins to break down. It is through this correlation breakdown that we can measure both the crack length and the crack growth rates. Both measurements are useful and necessary in in order to calculate fracture toughness.

\section{Sample geometry}

One of the principle drivers of this work was the limited availability of the materials used in this study. Initial tensile samples were machined having a gage dimension of $14 \mathrm{~mm}$ length $\times 3 \mathrm{~mm}$ width and $3 \mathrm{~mm}$ thickness. It was possible to measure the Poisson's ratio of these tensile samples using the DIC technique described above. As a result of preliminary tests it was determined that a test geometry that would slow the crack growth rate was necessary. The sample geometry was chosen based on work of Sammis et al. [1] and Plaisted, et al. [8]. In this case a rectangular sample with a center hole was used as the new test sample. It will be referred to as the "compression-fracture" (CF) sample throughout this paper. The nominal dimensions of the test sample are $25 \mathrm{~mm}$ in length or height $(\mathrm{H})$ by $9 \mathrm{~mm}$ width $(\mathrm{W})$ by the available material thickness $(\sim 4 \mathrm{~mm}$ for the DAP samples and $9 \mathrm{~mm}$ for the EBC material). A $3 \mathrm{~mm}$ diameter hole was machined in the sample centered on the length and width as shown in Fig. 1.

\section{Experimental evaluation}

Initially, the intention of this geometry was to generate crack growth in the test sample that is slow enough to capture images during deformation in order to measure crack growth rates. Early experiments showed that the load displacement curves do not provide any indication

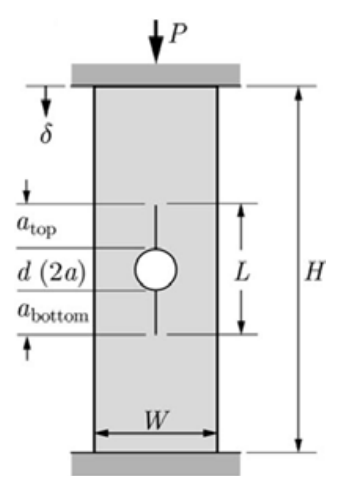

Figure 1. Schematic of the compression fracture test geometry.

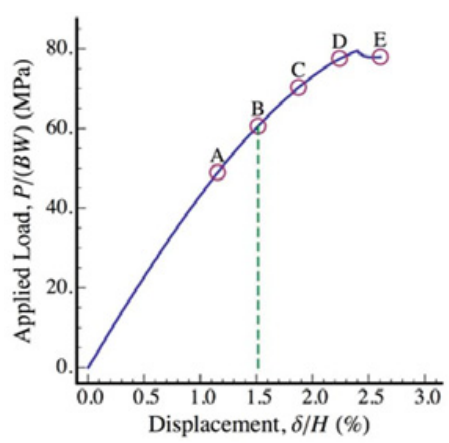

Figure 2. Example load displacement curve for DAP with indicators that correspond to sequential strain and correlation maps in the next figure. Location B is the moment when the correlation value begins to change rapidly.

of damage initiation. This is actually a positive as it means that the onset of cracking and crack growth is a graceful process for this geometry. However, it also makes it necessary to compare the DIC image evaluation with the load displacement data. An example plot of the load displacement curve (Fig. 2) for DAP is shown with indicators showing times when corresponding strain field maps are presented (Fig. 3). Using the technique to determine the correlation coefficient it is possible to define the location and time (displacement) when damage initiates. Images corresponding to point " $\mathrm{B}$ " give an indication of the onset of damage (as seen in the correlation maps) with further crack growth progressing to and beyond position "E". By analyzing all images using this technique it is possible to observe that there are two dominant cracks present, one propagating upward and the other down. This methodology is used to generate unique correlation and strain maps for every frame of the video that captured the test, thus, providing information about crack location, length, and growth rate.

It is necessary to elaborate on the technique used to determine the initiation and boundary of the cracks. This is done first by analyzing the maximum correlation value as it evolves during a test (Fig. 4). This plot shows a relatively flat, unchanging, value during the early part of the test, typically associated with the elastic loading of the sample. At the time when a crack initiates and begins to grow the correlation value also increases rapidly. The correlation value where this transition occurs is called the critical correlation coefficient. This coefficient will be used 


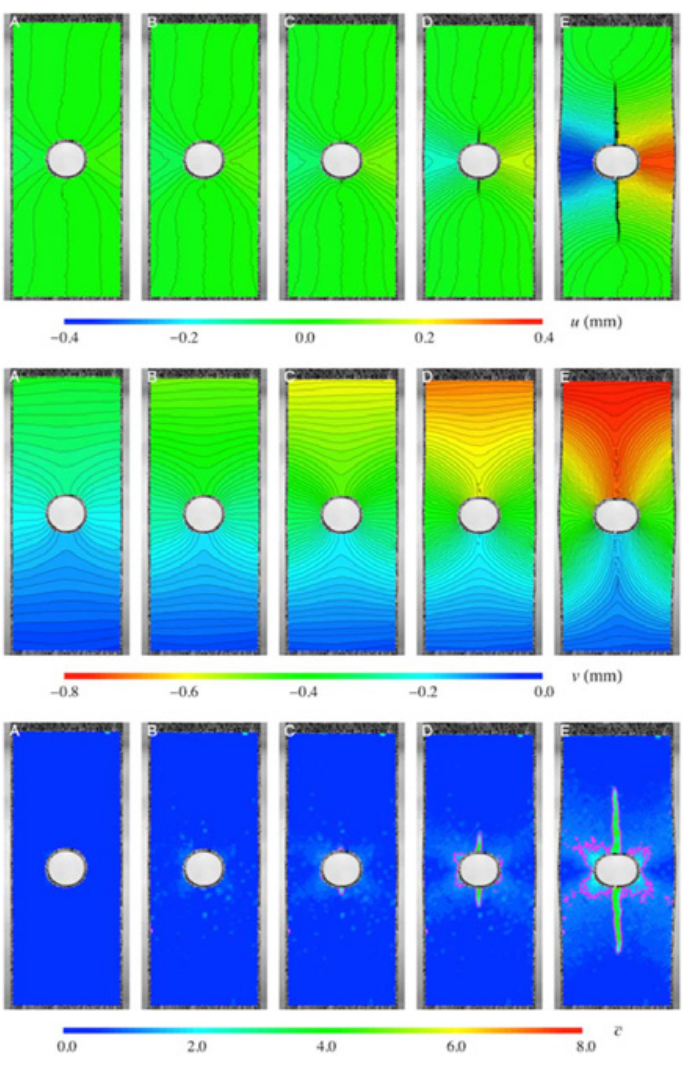

Figure 3. Images, marked with corresponding letters, associated with the moments in time indicated in Fig. 3. The top row of images is for the horizontal displacement fields, $u$. The middle row is for vertical displacement fields, $v$, and the bottom row are maps of the correlation coefficient.

to track the boundary of the "damaged" region showing where the crack is located and the rate at which it grows for the entire duration of the test. The maximum correlation value is not specifically related to a single point but once a crack is initiated the correlation value of that point will increase rapidly and this curve can be thought of as an accurate representation of the correlation coefficient at the damage initiation site. Typically, the maximum correlation value is associated with the region, if not exact position, of the onset of damage and the perimeter of the damage region can be traced when the value of correlation exceeds the critical correlation value.

The crack does not grow uniformly in both directions during the test (Fig. 5). The load versus displacement and the crack length versus displacement as analyzed from the correlation map (Fig. 3) shows this non-uniform crack growth.

\section{Fracture toughness calculation}

\subsection{Semi-empirical solution}

The fracture toughness, or critical stress intensity factor for the compression-fracture specimen is derived from the following equation. It has been modified to reflect the fact that the crack lengths may be different as they propagate from the center hole toward the top and bottom of the test sample. In this case the initial hole diameter is

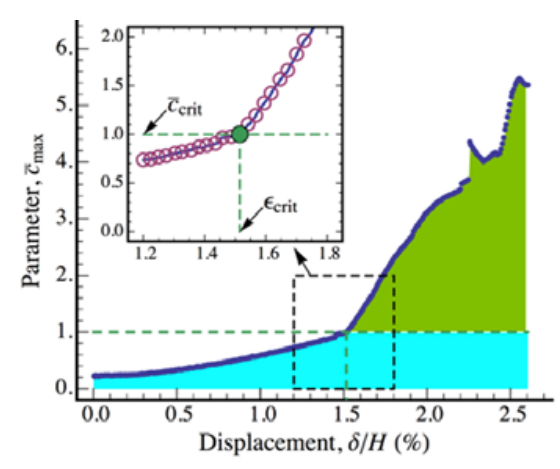

Figure 4. Plot of the correlation coefficient versus time. The inset shows how the critical correlation coefficient is determined. This plot is unique for each sample tested.

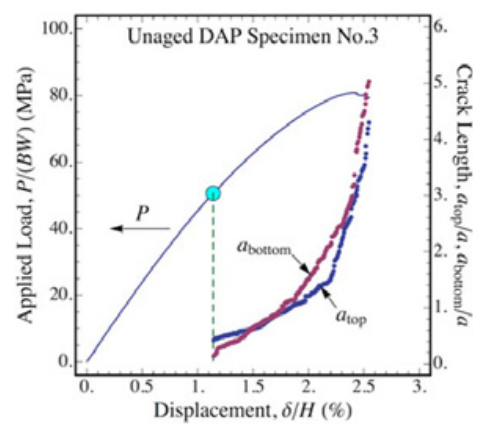

Figure 5. Plot of the applied load and crack length versus displacement with the upper and lower crack being individually represented.

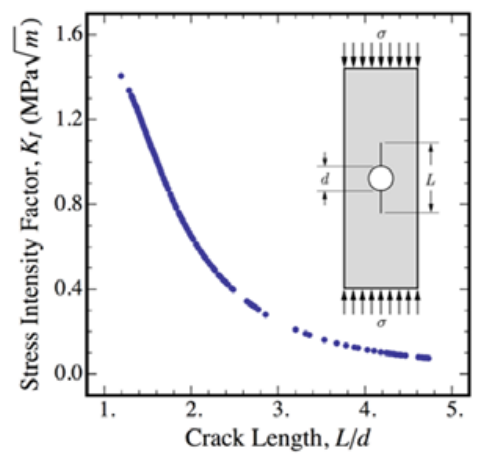

Figure 6. Example of the critical stress intensity factor for DAP using the compression fracture geometry.

$a$, the stress is the far field stress and is found by dividing the applied load by the loading area (width $x$ thickness). $a_{\text {top }}$ and $a_{\text {bottom }}$ are the crack length propagating from the top and bottom of the hole respectively.

$$
\left.K_{\mathrm{IC}}=-\left(\frac{a_{\mathrm{top}}+a_{\text {bottom }}}{2 a}\right)^{1 / 2}\left\{\frac{1.1}{\left(1+\frac{a_{\text {top }}+a_{\text {bottom }}}{2 a}{ }^{3}\right.}\right)\right\} \sigma \sqrt{\pi a} .
$$

Using the equation above a calculation of the stress intensity factor as a function of crack length was calculated assuming that the stress applied is equal to the applied load divided by the width and thickness of the sample at the contact surface (Fig. 6). The solution for the stress intensity 


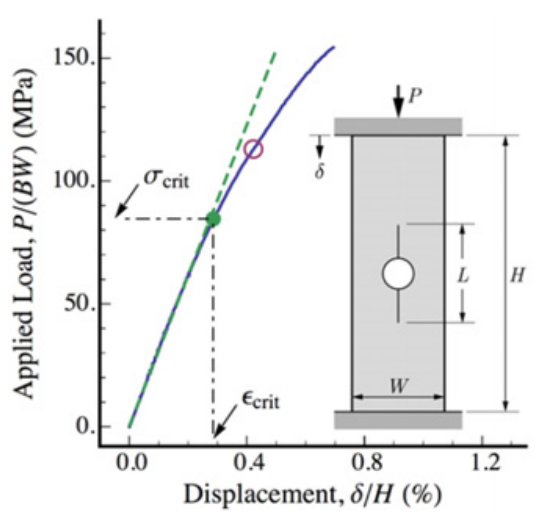

Figure 7. Comparison plot of the critical stress intensity for several experiments for un-aged and aged DAP.

factor is a semi-empirical in formula used to calculate the fracture toughness on a PMMA plate[1]. We have applied the technique as best we could to analyze our test results.

It should be noted that this experimental technique does not give a single value for the stress intensity factor. It does, however, show how crack growth and mechanical response to loading are correlated. Also, because the stress intensity factor is so closely tied to crack growth our hypothesis that this property is most sensitive to material change is likely correct.

It is important to remember that there are contributing factors that influence the initiation of fracture in all materials like those presented here. The factor that most greatly influences crack initiation is the finish quality of the hole. A very fine finish will likely cause initiation to be very high, whereas a rough finish is likely to improve the repeatability of the experiments. In the experiments presented here there was no specification made on the hole finish quality. This may be a contributing factor in the magnitude of the initial fracture toughness value.

\subsection{Linear elastic fracture mechanics solution}

In this section, we will apply Linearly Elastic Fracture Mechanics (LEFM) to further analyze the experimental data and extract the unique solution of fracture toughness for a given material. The definition of critical stress, $\sigma_{\text {crit }}$ and critical deformation, $\varepsilon_{\text {crit }}$, when crack initiation occurs, is shown using solid symbol in Fig. 7. We see that prior to the critical moment, the applied load, $P$, varies linearly as a function of the deformation, $\delta$, as indicated by the dashed line. The deviation from the linearity starts near the critical moment, which suggests that the apparent nonlinearity between applied load, $P$, and the deformation, $\delta$, is the result of the changing boundary or the motion of the growing crack. Therefore, all material elements within the specimen, except those very close to the crack tip, only experience elastic deformation. As a result, Linearly Elastic Fracture Mechanics (LEFM) theory can be applied to analyze the experimental data.

The displacement field measurement, obtained using DIC, over the surface of the specimen forms the basis of our data analysis. Consider a particular moment during the compression of a specimen, as shown in Fig. 8a, where two tensile cracks have initiated and extended. For each

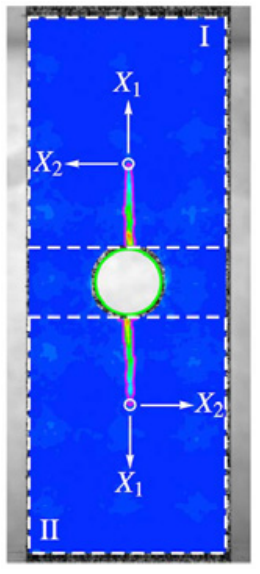

a)
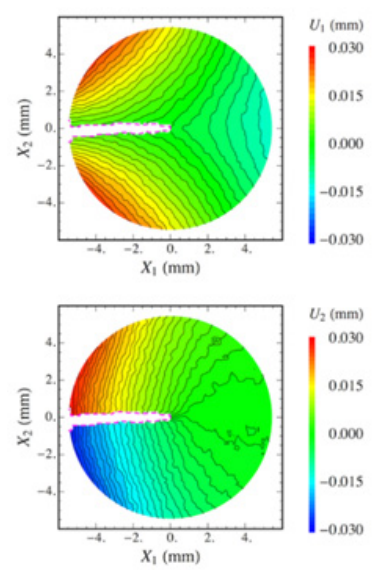

b)
Figure 8. a) Partitioning of the cracks propagating from the center hole, and b) strain maps in the "new" coordinate system for one end of the test sample.

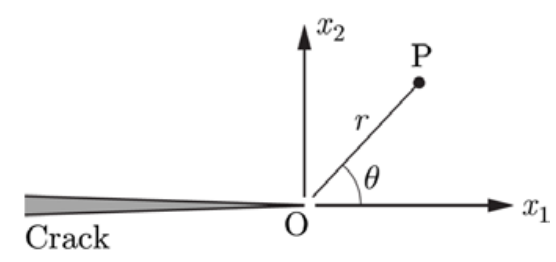

Figure 9. Crack-tip coordinate system.

tensile crack, we choose a coordinate system $\left(X_{1}, X_{2}\right)$ originating at the crack tip, determined according to our experimental scheme, the $X_{1}$-axis is parallel to the loading axis, or in the crack growth direction, and the $X_{2}$-axis is perpendicular to the loading axis. A rectangular region that surrounds each tensile crack is identified with (I and II). The displacement field surrounding the tensile crack at one end of the sample is shown in Fig. 8b, where $U_{1}$ is the component of strain (displacement) along the $X_{1}$-direction and $U_{2}$ is the component of strain (displacement) along the $X_{2}$-direction. We also assign that (wording) $\left(U_{1}=U_{2}=0\right)$ at the origin of the $\left(X_{1}, X_{2}\right)$ coordinate system.

For extracting crack tip parameters, a mathematical representation of the displacement field surrounding the crack is needed, and such a representation is usually expressed in the crack-tip coordinate system, as shown in Fig. 9. The $\left(x_{1}, x_{2}\right)$ system is originated at the crack tip and the $x_{1}$-axis is tangent to the crack at the crack tip and $x_{2}$ axis is perpendicular to the crack.

According to the asymptotic analysis of the elastic fracture mechanics [9], the displacement field can be represented in the asymptotic form,

$$
\begin{aligned}
& u(r, \theta)=\Sigma_{n=1}^{\infty} \frac{A_{n} r^{n / 2}}{2 \mu \sqrt{2 \pi}}\left\{\kappa \cos \frac{n \theta}{2}-\frac{n}{2} \cos \left(\frac{n}{2}-2\right) \theta\right. \\
& \left.+\left[\frac{n}{2}+(-1)^{n}\right] \cos \frac{n \theta}{2}\right\}+\Sigma_{n=1}^{\infty} \frac{B_{n} r^{n / 2}}{2 \mu \sqrt{2 \pi}} \\
& \quad \times\left\{\kappa \sin \frac{n \theta}{2}-\frac{n}{2} \sin \left(\frac{n}{2}-2\right) \theta+\left[\frac{n}{2}-(-1)^{n}\right] \sin \frac{n \theta}{2}\right\},
\end{aligned}
$$




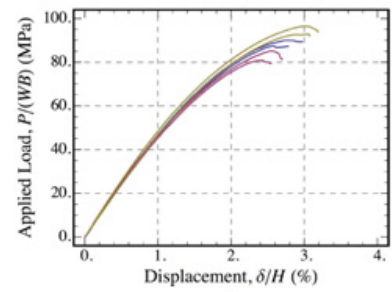

a)

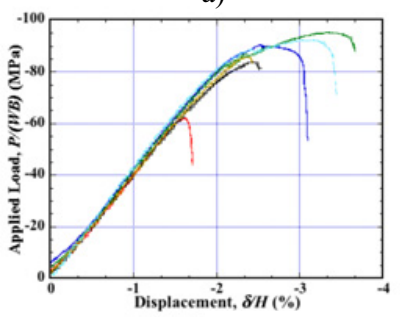

c)

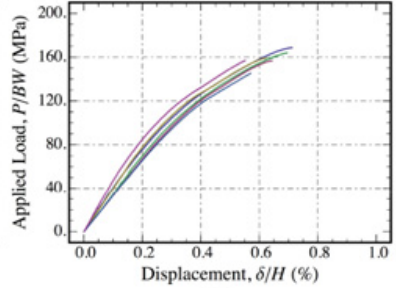

b)

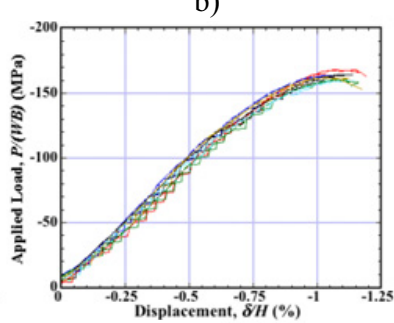

d)
Figure 10. Typical load displacement curves for a) DAP, low rate, b) $\mathrm{EBC}$, low rate, c) DAP high rate and d) $\mathrm{EBC}$ high rate.

$$
\begin{aligned}
& v(r, \theta)=\Sigma_{n=1}^{\infty} \frac{A_{n} r^{n / 2}}{2 \mu \sqrt{2 \pi}}\left\{\kappa \sin \frac{n \theta}{2}-\frac{n}{2} \sin \left(\frac{n}{2}-2\right) \theta\right. \\
& \left.-\left[\frac{n}{2}+(-1)^{n}\right] \sin \frac{n \theta}{2}\right\}+\Sigma_{n=1}^{\infty} \frac{B_{n} r^{n / 2}}{2 \mu \sqrt{2 \pi}} \\
& \times\left\{-\kappa \cos \frac{n \theta}{2}-\frac{n}{2} \cos \left(\frac{n}{2}-2\right) \theta+\left[\frac{n}{2}-(-1)^{n}\right] \cos \frac{n \theta}{2}\right\} .
\end{aligned}
$$

Where $(r, \theta)$ is the polar coordinates of a point, $\mu$ is the shear modulus of the material, and parameter $\kappa$ is related to the Poisson's ratio $v$ through

$$
\kappa=\left\{\begin{array}{l}
\frac{3-v}{1+v}, \text { for plane stress, } \\
3-4 v, \text { for plane strain. }
\end{array}\right.
$$

$A_{n}$ and $B_{n}$ are the coefficients of the asymptotic expansion and they cannot be determined by the asymptotic analysis itself but by the far-field conditions. In particular, for the leading term, $n=1$, we have $A_{\mathrm{I}}=K_{\mathrm{I}}$ and $B_{\mathrm{I}}=K_{I I}$, where $K_{I}$ and $K_{I I}$ are the stress intensity factors at the crack tip.

The asymptotic representation for a dynamically growing crack will be used to extract the dynamic stress intensity factor, or the dynamic fracture toughness, at the crack tip. Such an asymptotic representation can be found in $[10,11]$.

\section{Experimental results}

Typical load displacement responses for the two materials in this study are shown Fig. 10. From these plots it can be concluded that the test results are reproducible with acceptable differences in their loading behavior for all loading rates. These results also indicate that failure occurs at nominally the same load level independent of rate (with one exception). The principle difference between the low rate and high rate tests is seen in the strain to failure. In

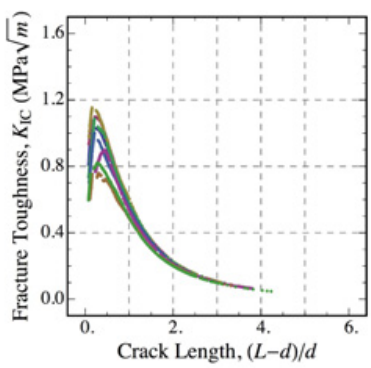

a)

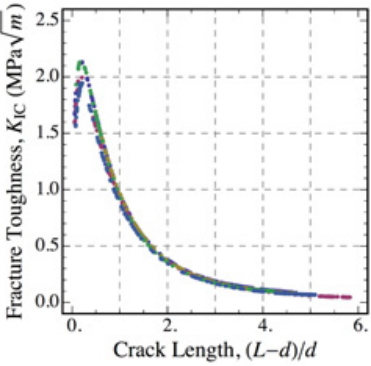

b)
Figure 11. Plots of the semi-empirical solution for fracture toughness for a) DAP and b) EBC.

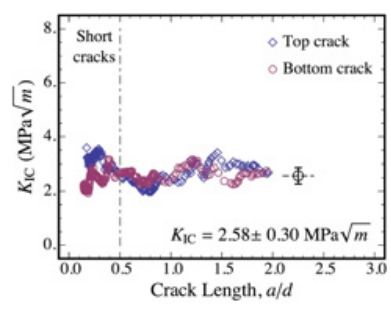

a)

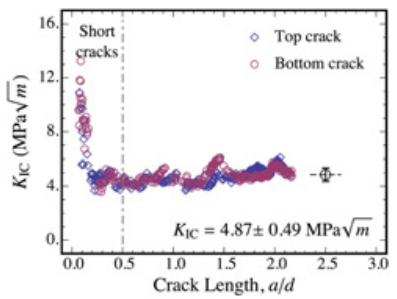

b)
Figure 12. Example of LEFM solution for slow crack growth $\left(\mathrm{K}_{1 \mathrm{C}}\right)$ in a) DAP and b) EBC.

general there is an increase in the strain to failure as the loading rate increased. This is consistent with other high stiffness, low ductility materials.

The crack velocity was measured for the different materials over the loading rates and was found to be somewhat variable. For the EBC material, the crack velocity was fairly constant through most of the deformation but slowed during the last $\sim 15 \%$ of each test. For the low loading rate test the crack velocity averaged $0.18 \mathrm{~mm} / \mathrm{s}$ and at the higher loading rate it was $\sim 2.5 \mathrm{~m} / \mathrm{s}$. The DAP material has a crack growth behavior that increases with displacement for the duration of the test (see Fig. 5).

A plot showing with the results of several tests using the semi-empirical solution for both materials shows reasonably good reproducibility (Fig. 11), if not the well understood material property definition for fracture toughness.

Typical plots for the LEFM solution for the low loading rate tests are presented below (Fig. 12). These plots provide insight into the development of fracture toughness. If the crack is arrested the value of $\mathrm{K}_{1 \mathrm{C}}$ increases, when it is growing quickly, $\mathrm{K}_{1 \mathrm{C}}$ drops. Uniform crack growth reduces the magnitude of the scatter and there is a minimum crack length for uniform crack growth on the order of the initial hole radius.

Uncertainties in the value for fracture toughness calculated using LEFM come from two sources. First, the scatter found within the analysis of a single test, and second, sample to sample variation. It is thought that, like in metals, a representative sample size is necessary to randomize the material being tested that will produce more uniform test results. At this point this is conjecture with out experimental support. 


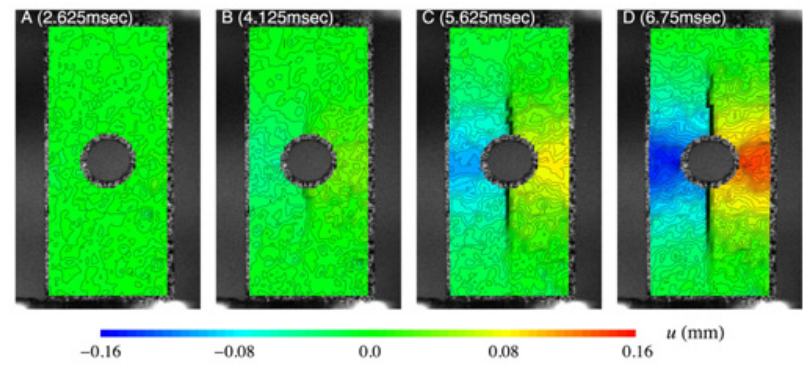

Figure 13. Displacement field map for EBC showing crack position as a function of time.

The challenge of the high strain rate testing is the resolution of the high speed camera system used. The system available for these experiments was only 320 pixel by 260 pixel. Unfortunately this made the determination of the crack location somewhat speculative and for the experiments conducted here the location of the crack was best determined from the $u$ displacement field (Fig. 13) instead of the correlation field that was used in the earlier analysis.

The image quality made it challenging to generate the correlation filed maps that are essential in tracking precisely the location of the crack tip. Since this is critical information in determining the fracture toughness the calculation for the fracture toughness using this technique will be postponed until a camera with a higher resolution can be found for these experiments.

\section{Conclusions}

The compression-fracture test geometry is suitable for measuring the fracture toughness of the materials in question and coordinated testing and evaluation techniques using high speed imaging, digital image correlation, and standard data acquisition from the load frames allowed for a calculation of fracture toughness using the semiempirical formula and LEFM with the only limitation of the analysis being subject to the capabilities of the equipment available.

The analysis technique to understand the fracture toughness of brittle and quasi-brittle materials was developed in this work. One of the benefits of using image capture and DIC is that it allows for re-evaluation of the test at any time in the future since the entire test is recorded for evaluation. A scheme for quantifying the extent of damage and cracking was also employed to determine the moment of crack initiation and the history of crack growth. This information was used to calculate the fracture toughness once the images were fully characterized.

The both materials exhibited easily traceable cracks and easily measureable crack growth rates that allowed for consistent calculation of the fracture toughness using the compression fracture test. A comparison fracture processes between the two materials studied indicates that the crack growth rate in the DAP material gradually increases, whereas, in the EBC material the growth rate is fairly uniform at the beginning of the test and gradually decreases near the end of the test due to loading constraints inherent in the test geometry.

The results for the stress intensity factor for the semiempirical solution has many constraints and assumptions which may not be correct, however, it does provide a straight forward comparison tool that will likely show material changes if they are present and significant. The mathematics and analysis scheme for LEFM solution has just recently been developed and the results shown here are for the first set of samples analyzed using this technique. One of the benefits of this technique is that the development of the crack and calculation of fracture toughness are tied together during this analysis. Therefore the value determined for $\mathrm{K}_{1 \mathrm{C}}$ can be averaged over a test with non-uniform crack extension at the beginning or end of the test removed from the solution.

The solution for the dynamic fracture toughness using LEFM will require using a modification to Eqs. (2) and (3) that are described by Freund [11]. It was possible to predict the approximate location of the crack from the displacement fields and therefor an approximation of the crack velocity. The test geometry is suitable for high rate tests, but a system with high resolution at high framing rates is necessary to determine the crack location.

Los Alamos National Laboratory is operated by Los Alamos National Security, LLC, for the National Nuclear Security Administration of the U.S. Department of Energy under contract DE-AC52-06NA25396.

\section{References}

[1] C. G. Sammis and M.F. Ashby, Acta Metall., 34 (3) pp 511-526 (1986)

[2] Y. Huang, C. Liu, M.G. Stout Acta mater 44 (3) pp 1223-1232 (1996)

[3] C. Liu, Y. Huang, M.L. Lovato, M.G. Stout, I J Fract, 87 (3) pp 241-263 (1997)

[4] R. Bedsole, H.V. Tippur. J Eng Mat and Tech Conference Proceedings 135 (3) pp 1-11 (2013)

[5] K.C. Jajam, H.V. Tippur, Composites Part B, Eng 43 (8) pp 3467-3481 (2012)

[6] K.C. Jajam, M.M. Rahman, M.V. Hosur, H.V. Tippur, Composites Part A, Appl Sci \& Mfg 59, pp 57-69 (2014)

[7] M.A. Sutton, S.R. McNeill, J.D. Helm, Y.L. Chao, (2000) Advances in Two-Dimensional and ThreeDimensional Computer Vision, Topics Appl. Phys. 77, pp 323-372 (2000)

[8] T.A. Plaisted, A.V. Amirkhizi, S. Nemat-Nasser, I J Fract, 141, pp 447-457 (2006)

[9] J.R. Rice, Fracture - An Advanced Treatise, Vol. II, H. Liebowitz (ed.), Academic, New York, pp. 191308, 1968

[10] L.B. Freund, Dynamic Fracture Mechanics, Cambridge University Press, New York, 1990

[11] C. Liu and A.J. Rosakis, Journal of Elasticity, 35, pp. 27-60, 1994 